\title{
Improved Sequence Learning with Subthalamic Nucleus Deep Brain Stimulation: Evidence for Treatment-Specific Network Modulation
}

\author{
Hideo Mure, ${ }^{1}$ Chris C. Tang, ${ }^{1}$ Miklos Argyelan, ${ }^{1}$ Maria-Felice Ghilardi, ${ }^{2}$ Michael G. Kaplitt, ${ }^{3}$ Vijay Dhawan, ${ }^{1}$ \\ and David Eidelberg ${ }^{1}$ \\ ${ }^{1}$ Center for Neurosciences, The Feinstein Institute for Medical Research, Manhasset, New York 11030, 2Department of Physiology and Pharmacology, City \\ University of New York Medical School, New York, NY 10031, and ${ }^{3}$ Department of Neurological Surgery, Weill Cornell Medical College, New York, New \\ York 10065
}

We used a network approach to study the effects of anti-parkinsonian treatment on motor sequence learning in humans. Eight Parkinson's disease (PD) patients with bilateral subthalamic nucleus (STN) deep brain stimulation underwent $\mathrm{H}_{2}{ }^{15} \mathrm{O}$ positron emission tomography (PET) imaging to measure regional cerebral blood flow ( $\mathrm{rCBF}$ ) while they performed kinematically matched sequence learning and movement tasks at baseline and during stimulation. Network analysis revealed a significant learning-related spatial covariance pattern characterized by consistent increases in subject expression during stimulation $(p=0.008$, permutation test). The network was associated with increased activity in the lateral cerebellum, dorsal premotor cortex, and parahippocampal gyrus, with covarying reductions in the supplementary motor area (SMA) and orbitofrontal cortex. Stimulation-mediated increases in network activity correlated with concurrent improvement in learning performance $(p<0.02)$. To determine whether similar changes occurred during dopaminergic pharmacotherapy, we studied the subjects during an intravenous levodopa infusion titrated to achieve a motor response equivalent to stimulation. Despite consistent improvement in motor ratings during infusion, levodopa did not alter learning performance or network activity. Analysis of learning-related $\mathrm{rCBF}$ in network regions revealed improvement in baseline abnormalities with STN stimulation but not levodopa. These effects were most pronounced in the SMA. In this region, a consistent $\mathrm{rCBF}$ response to stimulation was observed across subjects and trials $(p=0.01)$, although the levodopa response was not significant. These findings link the cognitive treatment response in PD to changes in the activity of a specific cerebello-premotor cortical network. Selective modulation of overactive SMA-STN projection pathways may underlie the improvement in learning found with stimulation.

\section{Introduction}

Parkinson's disease (PD) can affect cognitive functioning, even at its earliest clinical stages (Williams-Gray et al., 2007; Aarsland et al., 2009; Kehagia et al., 2010). Mild cognitive deficits in early PD typically involve visuomotor processing, working memory, and aspects of executive performance (Levin and Katzen, 2005; Aarsland et al., 2010). Motor sequence learning, which encompasses elements of these cognitive functions, has been studied extensively in human subjects (Doyon, 2008; Orban et al., 2010), and mild impairment of task performance has been noted in early-stage PD patients (Nakamura et al., 2001; Ghilardi et al., 2003; Carbon and Eidelberg, 2006;

\footnotetext{
Received Aug. 11, 2011; revised Nov. 14, 2011; accepted Nov. 22, 2011.

Author contributions: D.E. designed research; M.-F.G., M.G.K., and V.D. performed research; H.M., C.C.T., M.A., and M.-F.G. analyzed data; H.M., C.C.T., and D.E. wrote the paper.

This work was supported by National Institutes of Health/National Institute of Neurological Disorders and Stroke Grants R01 NS 35069 and P50 NS 071675 (D.E.). The content is solely the responsibility of the authors and does not necessarily represent the official views of the NINDS or the NIH. We thank Dr. Thomas Chaly for radiochemistry support and Claude Margouleff for technical assistance. Special thanks to Toni Fitzpatrick for valuable help with copyediting.

Correspondence should be addressed to Dr. David Eidelberg, Center for Neurosciences, The Feinstein Institute for Medical Research, 350 Community Drive, Manhasset, NY 11030. E-mail: david1@nshs.edu.

DOI:10.1523/JNEUROSCI.4331-11.2012

Copyright $\odot 2012$ the authors $\quad 0270-6474 / 12 / 322804-10 \$ 15.00 / 0$
}

Wilkinson et al., 2009; Carbon et al., 2010b). In this vein, the effects of anti-parkinsonian interventions on cognitive functioning have been found generally to be independent of motor benefit (Cools et al., 2006; Frank and Claus, 2006; Kehagia et al., 2010). Dopamine replacement for the treatment of PD motor symptoms is associated with subtle changes in sequence learning performance assessed at the group mean level (Feigin et al., 2003; Ghilardi et al., 2007). Indeed, recent evidence suggests that individual differences in the cognitive response to levodopa relate to baseline performance capacity (Argyelan et al., 2008; Mattis et al., 2011). This contrasts with the consistent improvement in sequence learning performance observed in medication refractory PD patients treated with internal globus pallidus (GPi) deep brain stimulation (DBS) (Fukuda et al., 2002; Carbon et al., 2003). Although the subthalamic nucleus (STN) DBS procedure that is in routine use has been found to have minimal impact on executive functioning (Parsons et al., 2006; Williams et al., 2011), little is known of its specific effects on motor sequence learning performance. Although levodopa treatment and STN stimulation have both been found to have similar effects on the expression of PD motor networks (Asanuma et al., 2006), it is not known how these interventions affect the neural pathways that mediate cognitive functioning in this disorder. 
Table 1. Subject characteristics

\begin{tabular}{lllll}
\hline & \multicolumn{2}{l}{ Parkinson's disease } & $\begin{array}{l}\text { Healthy } \\
\text { control }\end{array}$ \\
\cline { 2 - 5 } & OFF & DBS & LD & 8 \\
\hline Subject number & 8 & & $52.7 \pm 13.7$ \\
Age (years, mean \pm SD) & $55.8 \pm 8.5^{a}$ & & $6: 2$ \\
Male/female & $6: 2$ & & \\
Disease duration (years) & $12.1 \pm 4.6$ & & & \\
UPDRS (motor) & $24.4 \pm 5.5$ & $17.6 \pm 4.8^{* *}$ & $16.2 \pm 3.8^{* *}$ & \\
Random reaction time (ms) & $436 \pm 136$ & $347 \pm 57^{*}$ & $353 \pm 55^{*}$ & $216 \pm 17$ \\
Retrieval index & $11.8 \pm 8.9$ & $23.1 \pm 14.2^{*, \dagger}$ & $11.4 \pm 10.7$ & $31.9 \pm 20.2$ \\
\hline
\end{tabular}

${ }^{a}$ Mean \pm SD.

${ }^{b}$ UPDRS motor ratings (items $19-31$ ).

${ }^{*} p<0.05$, ${ }^{* *}$, relative to baseline (Tukey-Kramer HSD).

${ }^{\dagger} p<0.05$, relative to levodopa treatment (Tukey-Kramer HSD).

In the current study, we used a combined psychophysical and functional imaging strategy to evaluate the changes in sequence learning performance and associated network activity that occur during STN stimulation, which were then compared with those observed during levodopa treatment. PD patients and healthy control subjects were scanned with ${ }^{15} \mathrm{O}$-labeled water $\left(\mathrm{H}_{2}{ }^{15} \mathrm{O}\right)$ positron emission tomography (PET) while performing kinematically equivalent motor sequence learning and execution tasks (Nakamura et al., 2001; Ghilardi et al., 2003). The PD subjects performed these tasks in the untreated baseline condition, during STN stimulation, and again while receiving an intravenous levodopa infusion titrated to a comparable degree of clinical improvement. A within-group functional connectivity model (Habeck et al., 2005; Moeller and Habeck, 2006) was applied to identify a specific learning-related network in the imaging data acquired in the baseline and on-stimulation conditions. We compared the degree of network modulation achieved by STN stimulation and levodopa infusion in the same subjects. Additionally, treatment-mediated differences in network activity were correlated with concurrent changes in learning performance recorded during imaging.

\section{Materials and Methods}

Subjects

We studied eight right-handed PD subjects (six men and two women; age, $55.8 \pm 8.5$ years, mean \pm SD; Hoehn and Yahr Stage $2.5 \pm 0.4$ ) with bilaterally implanted STN DBS electrodes. The demographic features of these subjects are presented in Table 1. The participants exhibited no evidence of dementia or depression on neuropsychological testing conducted in the baseline untreated condition [estimated intelligence quotient (Nelson, 1982; Blair and Spreen, 1989), $121 \pm 7$; Beck Depression Inventory (Beck, 1987), $5 \pm 3$; Brief Test of Attention (Schretlen, 1989), $7 \pm$ 1; Hopkins Verbal Learning Test Trial 1-5, $20 \pm 6$; delayed recall, $4 \pm$ 4 (Brandt, 1991; Benedict et al., 1998); Symbol Digit Test (Smith, 1982), $33 \pm 8$; controlled oral word association (Benton et al., 1983), $44 \pm 15$ ]. There was no evidence of atrophy or other structural brain abnormalities on routine magnetic resonance imaging (MRI).

The subjects were scanned with $\mathrm{H}_{2}{ }^{15} \mathrm{O}$ PET in a single imaging session while they performed two kinematically matched reaching tasks (described below) in each of the following experimental conditions: (1) OFF: a baseline condition in which anti-parkinsonian medications were discontinued for at least $12 \mathrm{~h}$ beforehand and with stimulators turned off for at least $1 \mathrm{~h}$; (2) DBS: a treatment condition in which stimulation on the two sides (amplitude, $3.29 \pm 0.41 \mathrm{~V}$; pulse width, $67.5 \pm 12.5 \mu \mathrm{s}$; frequency, $176.7 \pm 20.4 \mathrm{~Hz}$ ) was optimized to achieve a clinically meaningful improvement in motor signs, defined as a reduction on Part III of the Unified Parkinson's Disease Rating Scale (UPDRS) (Fahn et al., 1987) of at least five points or $20 \%$ of baseline; and (3) LD: a treatment condition in which the subjects received a steady-state intravenous infusion of levodopa without stimulation (Feigin et al., 2003; Hirano et al., 2008).
In this condition, the levodopa infusion rate (mean of $1.13 \pm 0.41$ $\mathrm{mg} \cdot \mathrm{kg}^{-1} \cdot \mathrm{h}^{-1}$ ) was individually titrated to produce an improvement in motor UPDRS ratings equivalent to that achieved by stimulation without dyskinesia. Eight healthy volunteer subjects (six men and two women; age, $52.7 \pm 13.7$ years) served as controls for these experiments. These subjects underwent $\mathrm{H}_{2}{ }^{15} \mathrm{O}$ PET while performing the same set of tasks in a single imaging session.

\section{Behavioral testing}

Tasks. In each experimental condition, the subjects were scanned during the performance of a motor sequence learning task (LEARN) and a kinematically equivalent motor execution task (MOVE). The characteristics of these reaching tasks have been described in detail previously (Ghilardi et al., 2003; Argyelan et al., 2008; Ghilardi et al., 2009). Briefly, in performing the two reaching tasks, subjects moved a cursor on a digitizing tablet with the dominant right hand. Movements were out and back from a central starting position to one of eight radial targets displayed on the screen. In LEARN, the eight targets appeared in an unknown, pseudorandom order without repeating elements that was repeated over the $90 \mathrm{~s}$ trial block (SEQ). Subjects were informed that a sequence was to be presented. They were instructed to learn the sequence order while reaching for the targets, to anticipate successive targets, and to reach each target in synchrony with the tone. Sequences were different for each trial run. In MOVE, the eight targets appeared in a predictable counterclockwise (CCW) order. To reach the target in synchrony with the tone, subjects had to initiate movement before the target appeared. In addition to the reaching tasks, the subjects were also scanned in a nonmovement sensory-matched resting state (REST) in which they passively observed randomly appearing targets (Ghilardi et al., 2000).

To evaluate the effects of STN stimulation on sequence learning and associated brain networks, we designed the imaging experiments so that the order of the trials was randomized and counterbalanced across tasks (LEARN, MOVE) and treatment conditions (OFF, DBS). Because of the long-duration effect of levodopa on motor symptoms (Hauser and Holford, 2002), the infusion studies (LD) followed the DBS and OFF trials. Thus, the possibility existed that the observed differences in learning across experimental conditions, if any, were influenced by treatment order, with practice improving and/or fatigue worsening task performance during levodopa infusion relative to STN stimulation. To address this potential confound, we measured the random reaction time outside the scanner before each LEARN trial. To quantify this measure of perceptual motor processing speed, targets were presented in pseudorandomized, nonrepeating, and unpredictable order, with the subjects required to reach each target as soon as possible (Nakamura et al., 2001). Random reaction time values were measured separately in each of the three experimental conditions. As described below, this measure was used as an empiric criterion for target anticipation during learning performance in each condition (Ghilardi et al., 2003, 2009). The random reaction time measurements were also compared across conditions to determine whether order-dependent changes in motor processing speed (an index of general attentiveness) were evident in the data.

The order of task trials was randomized and counterbalanced within each of the three experimental conditions. Of note, the pace of the movements in LEARN and MOVE, represented by the intertone interval, was empirically adapted to the subject's motor ability in the baseline OFF state. This allowed us to fix the rate of movement across tasks (LEARN, MOVE) and conditions (OFF, DBS, LD). The intertone interval was $1.5 \mathrm{~s}$ in five of the eight PD subjects, $2.0 \mathrm{~s}$ in two of the subjects, and $1.83 \mathrm{~s}$ in one of the subjects. Similarly, we held movement size constant by adjusting the gain to a target extent of $1 \mathrm{~cm}$ for all subjects, tasks, and experimental conditions.

Performance measures. To quantify learning performance in each LEARN run, we computed the number of correct movements initiated below a threshold determined by the random reaction time (Nakamura et al., 2001; Ghilardi et al., 2003, 2009). This threshold was determined outside the scanner and separately quantified for each experimental condition. Movements initiated below this reaction time reflect anticipation and successful retrieval of previously acquired targets. The number of correctly anticipated targets in each cycle were summed defining a com- 
posite retrieval index that quantified task performance in each LEARN trial (Ghilardi et al., 2003; Argyelan et al., 2008; Ghilardi et al., 2009). The effects of experimental condition (OFF, DBS, LD) on motor disability (UPDRS motor ratings), perceptual motor processing speed (random reaction time), and learning performance (retrieval index) were assessed in separate one-way repeated-measures analysis of variance (RMANOVA) models; pairwise contrasts were assessed post hoc using Tukey's HSD tests. Additionally, psychophysical performance measures in each condition were compared with control values using two-tailed Student's $t$ tests. Contrasts were considered significant at $p<0.05$.

\section{Imaging studies}

Positron emission tomography. All subjects were scanned with $\mathrm{H}_{2}{ }^{15} \mathrm{O}$ PET at baseline (OFF) and in each of the two treatment conditions (DBS, LD) in a single $1 \mathrm{~d}$ imaging session (Argyelan et al., 2008). The subjects fasted overnight before imaging; anti-parkinsonian medications were discontinued at least $12 \mathrm{~h}$ beforehand. PET imaging was performed using the GE Advance tomograph (GE Healthcare) in 3D mode. In the baseline and treatment conditions, the subjects performed the SEQ and CCW tasks in randomized order using the dominant right arm. Four of the subjects performed the tasks twice in the three experimental conditions. Because of fatigue, the remaining subjects performed the tasks once per condition. Thus, 12 complete scan sets (i.e., pairs of SEQ and CCW scans from individual subjects studied in the OFF and DBS conditions) were available for pattern derivation (see below).

Ethical permission for these studies was obtained from the Institutional Review Board of The Feinstein Institute for Medical Research. Written consent was obtained from each subject after detailed explanation of the procedures.

Network analysis. Imaging data processing was performed using SPM5 (Institute of Neurology, London, UK). Standard preprocessing (realignment, spatial normalizing, smoothing with an isotropic Gaussian kernel $(10 \mathrm{~mm})$ in all directions) was conducted. To identify a specific functional brain network associated with STN stimulation effects on motor sequence learning, we applied a within-subject network modeling approach to the imaging data termed Ordinal Trends/Canonical Variates Analysis (OrT/CVA) (Habeck et al., 2005; Moeller and Habeck, 2006). The OrT/CVA algorithm is designed to identify specific spatial covariance patterns that increase in their expression across experimental conditions on a subject-by-subject basis while the relationships between brain regions remain constant (Carbon et al., 2010a; Mure et al., 2011). OrT/CVA differs from routine univariate analysis in that it requires that pattern expression values exhibit an "ordinal trend": the property of consistent change across conditions at the individual subject level (i.e., network activity) is required to increase monotonically in all or most of the subjects. As in groupwise spatial covariance analysis (Eidelberg, 2009; Habeck and Stern, 2010; Spetsieris and Eidelberg, 2011), large-scale networks are described in terms of the voxel loadings ("region weights") on each of the relevant principal component (PC) topographies. Likewise, the expression of a given pattern in each scan is quantified by a specific network activity value ("subject score"), the PC scalar multiplier for the subject in each task and experimental condition. The significance of networks resulting from OrT/CVA is assessed using nonparametric tests. In pattern derivation datasets, permutation tests of the relevant subject scores are used to confirm that the observed monotonic changes in pattern expression across conditions did not occur by chance. The reliability of the voxel loadings comprising the network topography itself is assessed using bootstrap resampling procedures (Efron and Tibshirani, 1994).

In the current study, OrT/CVA was used to characterize the effects of STN stimulation on learning-related cerebral blood flow at the network level. The computational algorithm was applied to the LEARN scans of the PD patients acquired in the OFF and DBS conditions. To minimize confounds stemming from the concurrent effects of anti-parkinsonian treatment on movement (as opposed to learning), we restricted the search for learning-related network topographies to the portion of the subject $X$ voxel space that was independent of motor activation. This was accomplished by defining a subspace relating to motor activation in the current patient and control sample. Specifically, in a previous study (Carbon et al., 2010a), we used OrT/CVA to identify a significant reproduc- ible movement-related spatial covariance pattern in motor execution (MOVE) and rest (REST) $\mathrm{H}_{2}{ }^{15} \mathrm{O}$ PET scan pairs from 18 healthy volunteer subjects. We then quantified the expression of this normal movement-related activation pattern (NMRP) in the MOVE and REST scans acquired in the individual PD patients in each of the three treatment conditions and the healthy control subjects that comprised the current dataset. Analysis of the resulting subject scores disclosed consistent MOVE $>$ REST increases in each group and condition $(p<0.003$, paired Student's $t$ tests) with minimal exceptions. Having demonstrated that the individual motor activation responses were present within the NMRP subspace, we conducted the OrT/CVA search for learning-related patterns in the subspace orthogonal to that network, i.e., that which was independent of movement-related subject $\times$ voxel variability in the data.

The resulting OrT/CVA networks were considered significant if a consistent monotonic trend ( $p<0.01$, permutation test, 1000 iterations) was demonstrable for pattern expression values quantified in OFF/DBS scan pairs acquired during learning performance (i.e., increasing subject scores for DBS vs OFF computed in the LEARN scans). The corresponding spatial topographies were displayed at a voxel weight threshold of $Z=$ $2.81(p=0.005)$ with an extent cutoff of 50 voxels. Clusters present at this threshold were considered to represent significant regional "nodes" of the network if they were additionally found to be reliable by bootstrap resampling, as defined by an inverse coefficient of variation $>1.64$ ( $p<$ $0.05,1000$ iterations).

After network identification in OFF/DBS LEARN scan pairs, pattern expression was prospectively quantified in the MOVE scans of the PD patients acquired in these two experimental conditions and in the healthy control subjects. Network activity was additionally measured in the LEARN and MOVE scans of the PD patients acquired during levodopa infusion. As with network identification, prospective computation of pattern expression values in individual subjects/scans was conducted in the space orthogonal to the NMRP motor activation subspace (see above). Network values computed for each task/condition were $z$-transformed with respect to the corresponding healthy control values so that the normal (NL) group had a mean of 0 and an SD of 1. All OrT/CVA computations were performed using software freely available at http://www.nitrc.org/projects/gcva_pca.

Data analysis. Network scores computed in the baseline LEARN and MOVE scans of the PD patients were compared with those from the healthy control subjects. Group differences were evaluated using $2 \times 2$ RMANOVA with group (PD, NL) and task (LEARN, MOVE) as the between- and within-subject factors. For each subject, we additionally computed a learning-specific measure of network activity. This was defined as the difference in pattern expression computed in the LEARN and MOVE scans from that individual (i.e., the LEARN - MOVE differences in subject scores). The resulting value was correlated with the corresponding learning performance measure from the same subject. Correlation analysis was performed separately in each group by calculating Pearson's correlation coefficients. Network activity and task performance values from individual subjects with multiple LEARN runs were averaged for this analysis.

Similar statistical analyses were performed to quantify subject differences in network activity occurring during treatment and to correlate these measures with concurrent changes in task performance. Stimulation-mediated changes in pattern expression measured in LEARN and MOVE scans were assessed using paired Student's $t$ tests. Likewise, network values measured during stimulation (DBS) were compared with the corresponding normal values using Student's $t$ tests. Analogously, network values computed in LEARN and MOVE scans acquired during stimulation were compared with corresponding values from treatment scans acquired during levodopa infusion (LD). Network values computed during levodopa treatment were also compared with those from the healthy control subjects. For each intervention, we also correlated learning-specific network modulation during treatment with concurrent changes in task performance. As above, this was done by computing Pearson's product moment correlation coefficients.

\section{Nodal analysis}

After characterizing the effects of treatment at the network level, we evaluated the changes that occurred concurrently at each of the network 
A Motor Ratings

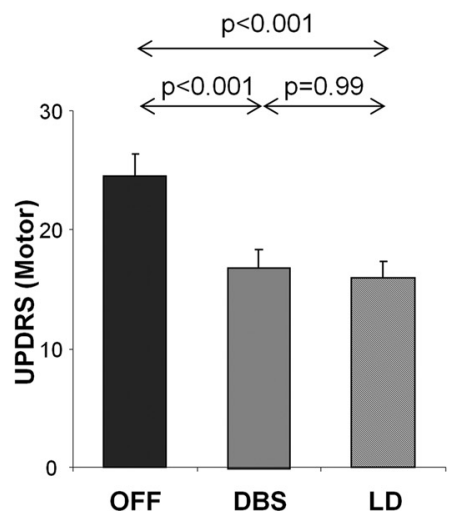

B

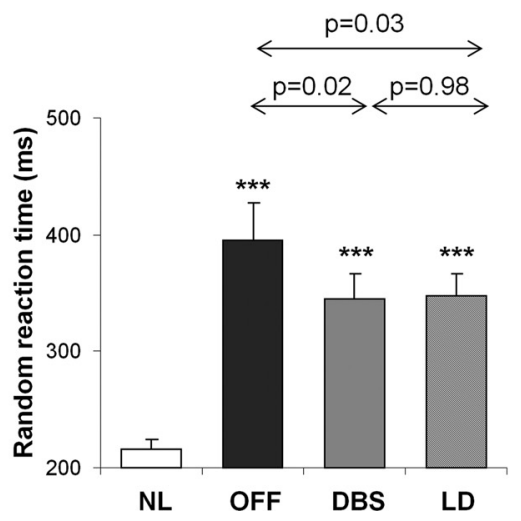

C

Learning Performance

Figure 1. Treatment-mediated changes in clinical motor ratings, reaction time, and retrieval index. $A$, Clinical motor ratings according to the UPDRS Part IIl in the PD subjects differed ( $p<0.001$, RMANOVA) across the three experimental conditions (see Materials and Methods). Relative to the untreated baseline condition, comparable degrees of motor improvement were observed during the treatment of motor symptoms with either STN stimulation or intravenous levodopa infusion ( $p<0.001$, Tukey-Kramer HSD). B, Random reaction time, a measure of perceptual motor processing speed, differed across conditions $(p<0.001)$, with comparable improvement in the two treatment conditions $(p<0.05$, relative to baseline). Nonetheless, this measure remained abnormally elevated in the PD patients regardless of treatment status (*** $p<0.001$, Student's $t$ tests). C, Retrieval index, a measure of sequence learning performance, also showed a significant difference across conditions $(p<0.01)$. However, unlike the motor ratings and reaction time measurements, learning performance selectively improved with STN stimulation $(p<0.01)$. Indeed, relative to healthy subjects, learning performance was reduced at baseline and in the levodopa treatment condition $\left({ }^{*} p<0.05\right)$.

nodes. This was done by measuring regional cerebral blood flow (rCBF) post hoc in volumes of interest (VOIs) corresponding to the significant clusters that defined the network topography (see above). Each node was represented by a spherical VOI (radius of $4 \mathrm{~mm}$ ) centered at the peak voxel of the corresponding cluster. Only nodes with significant changes in $\mathrm{rCBF}$ with STN stimulation ( $p<0.05$, paired Student's $t$ test) were selected for additional analysis. In these VOIs, $\mathrm{rCBF}$ values measured in the OFF, DBS, and LD conditions in the PD patients were compared with corresponding values from the healthy control subjects using Student's $t$ tests. Differences in rCBF values measured in the DBS and LD conditions were assessed separately for each node using paired Student's $t$ tests. The results were considered significant at $p<0.05$.

Finally, we used the rCBF data from each of the VOIs to explore the relative influence of the individual nodes on overall network behavior during treatment. Specifically, for each node, we determined the degree to which the effects of STN stimulation on rCBF (an index of local synaptic activity) were consistent across subjects and trials. This was done separately for each region by computing the number of trials (in proportion to the total) that violated the ordinal trend observed for the network as a whole. We hypothesized that network nodes exhibiting uniform rCBF responses to stimulation (i.e., with significantly fewer individual trials that violated the network-level trend) had closer functional connection to the DBS target than those that exhibited less consistent (i.e., more random) responses at the individual trial level. For the positive nodes (i.e., those characterized by network-related increases in activity during stimulation), trials exhibiting concurrent treatment-mediated declines in $\mathrm{rCBF}$ were considered to be at variance with the network trend and were classified as exceptions. Analogously, for the negative nodes (i.e., those with network-related decreases in activity during stimulation), trials with increasing $\mathrm{rCBF}$ during treatment were considered inconsistent with the network-level response and were counted as exceptions. Operationally, rCBF responses to stimulation at a given node were viewed as consistent if the frequency of exceptions in the individual trial data was lower than expected by chance. This possibility was evaluated using the binomial test and was considered significant for $p<0.05$ after Bonferroni's correction for multiple regional comparisons.

All statistical analyses were conducted using SPSS software, and the results were considered significant at $p<0.05$.
Table 2. Regions comprising the learning-related brain network

\begin{tabular}{|c|c|c|c|c|}
\hline \multirow[b]{2}{*}{ Brain region } & \multicolumn{3}{|c|}{ Coordinates $^{a}$} & \multirow[b]{2}{*}{ Zmax } \\
\hline & $x$ & $y$ & z & \\
\hline \multicolumn{5}{|l|}{ Increasing (DBS > OFF) } \\
\hline Right cerebellum (lobule VII) ${ }^{b}$ & -34 & -54 & -50 & $4.01^{* * *}$ \\
\hline Right parahippocampal gyrus (BA36) & -28 & 4 & -36 & $3.40^{* *}$ \\
\hline Left inferior parietal (BA40) & 44 & -26 & 42 & $2.91^{*}$ \\
\hline Left premotor cortex (BA6) & 32 & 20 & 58 & $2.75^{*}$ \\
\hline \multicolumn{5}{|l|}{ Decreasing (DBS < OFF) } \\
\hline Orbitofronal cortex (BA11) & 2 & 22 & -12 & $3.71^{* *}$ \\
\hline SMA & 0 & -18 & 68 & $2.72^{*}$ \\
\hline
\end{tabular}

${ }^{a}$ Montreal Neurological Institute standard space.

${ }^{b}$ According to the atlas of Schmahmann et al. (2000).

${ }^{*} p<0.005,{ }^{* *} p<0.001,{ }^{* * *} p<0.0001$ (at peak voxel).

\section{Results}

\section{Behavioral measures}

Mean \pm SD values for the clinical motor ratings, random reaction times, and learning performance measures for the $\mathrm{PD}$ patients in the OFF, DBS, and LD conditions, and for the healthy control subjects are presented in Table 1. UPDRS motor ratings (Fig. $1 A$ ) differed significantly across the three experimental conditions $\left(F_{(2,14)}=24.6\right.$, $p<0.001$, one-way RMANOVA), with improvement in clinical motor ratings during anti-parkinsonian treatment with either STN stimulation or levodopa infusion $(p<0.001$ relative to baseline, post hoc tests). Between-condition differences $\left(F_{(2,13)}=8.2, p<0.005\right)$ were also observed for random reaction time values (Fig. $1 B$ ), with significant reductions in this measure in both treatment conditions ( $p<0.05$ relative to baseline, post hoc tests). Sequence learning performance (Fig. $1 C$ ) also differed across conditions $\left(F_{(2,14)}=7.1, p<\right.$ $0.01)$. However, in contrast to the motor ratings and reaction time measures, sequence learning differed for the two interventions, with significantly better performance during STN stimulation relative to both levodopa and the untreated baseline condition $(p=0.01$, post hoc tests). Of note, there was no correlation between stimulation-related improvement in learning performance and the DBS parameters used during imaging $(p>0.34)$. In 


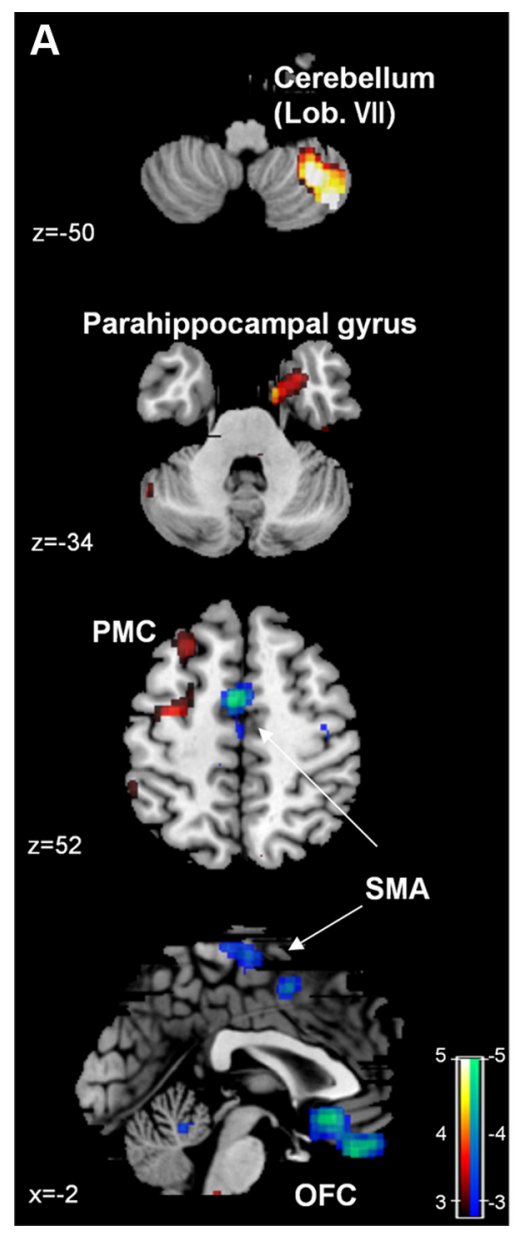

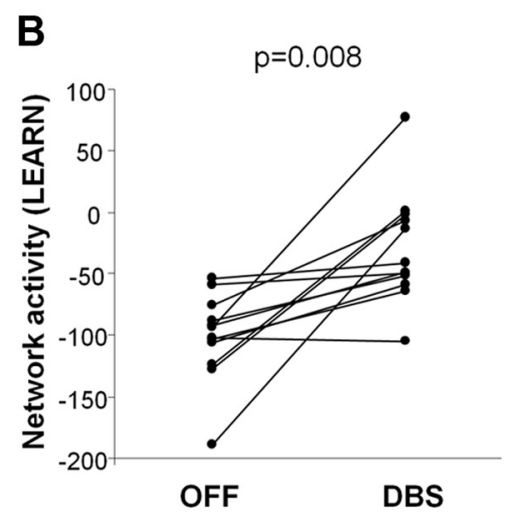

D

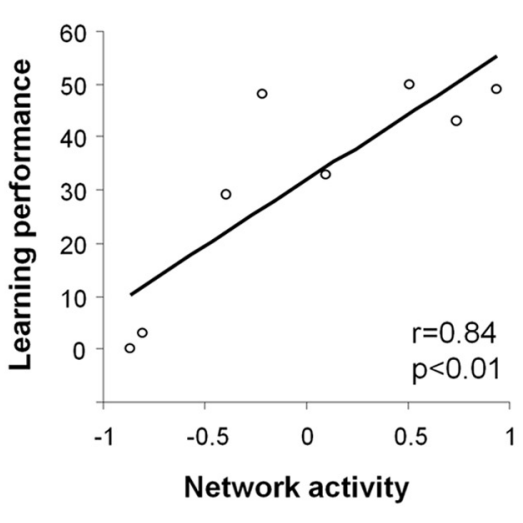

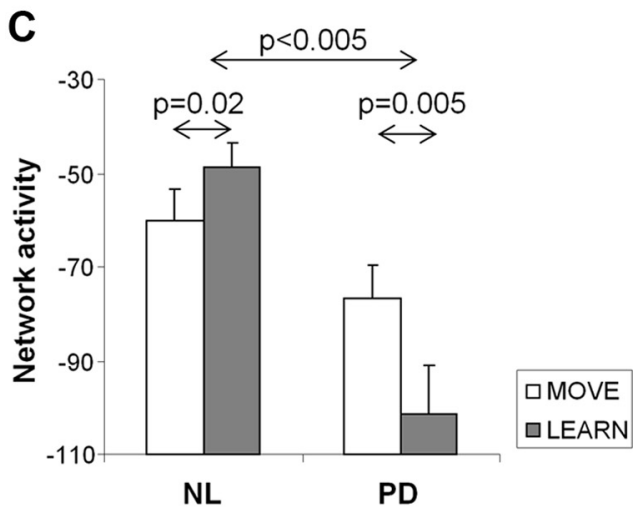

E

Parkinson's Disease

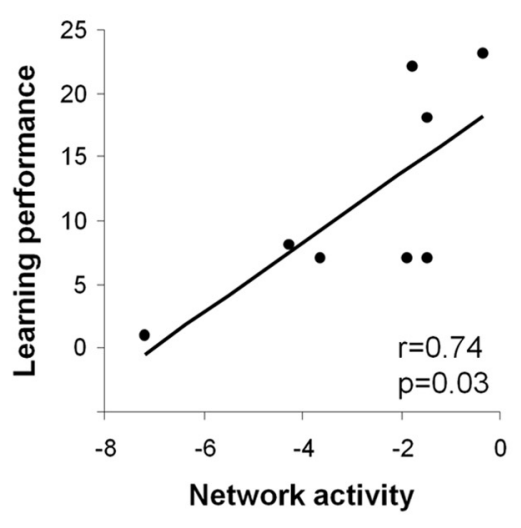

Figure 2. Effects of STN stimulation on network activity during motor sequence learning. $\boldsymbol{A}$, Spatial covariance pattern identified by OrT/CVA of $\mathrm{H}_{2}{ }^{15} \mathrm{OPET}$ scans acquired on- and off-STN stimulation during the performance of a motor sequence learning task (see Materials and Methods). The pattern was characterized by increasing activity (red) in the right cerebellum and parahippocampal gyrus and in the left premotor cortex (PMC) and inferior parietal region. These changes were associated with reductions (blue) in the orbitofrontal cortex (OFC) and the SMA. [The network map was overlaid on T1-weighted MR-template images. Voxel weights were thresholded at $|Z|=2.81, p<0.005$. The display represents regions that were demonstrated to be reliable $(p<0.05)$ on bootstrap resampling (1000 iterations).] B. Network activity increased during stimulation in 11 of 12 learning trials ( $p<0.008$, permutation test). C, In the NL control subjects, network activity during learning (gray bars) was higher ( $p<0.05$, RMANOVA) than during movement (white bars). In contrast, at baseline, the PD subjects exhibited reduced network activity measured during learning relative to movement $(p=0.005)$. Indeed, learning-specific network responses (i.e., differences between LEARN and MOVE subject scores; see Materials and Methods) differed significantly for PD and healthy control groups $(p<0.005) . \boldsymbol{D}, \boldsymbol{E}$, Network activity correlated with concurrent online measurements of learning performance in the healthy control subjects $(r=0.84, p<0.01)$ and in the PD patients scanned in the baseline condition $(r=0.74, p<0.05)$.

contrast to STN stimulation, learning performance measured during levodopa infusion did not differ from baseline values $(p=0.99)$. Indeed, target retrieval was abnormally low both at baseline and during levodopa treatment $(p<0.02$ compared with healthy control values, Student's $t$ tests), whereas learning performance measured during STN stimulation did not differ significantly from normal $(p=0.45)$.

\section{Network characterization}

A significant functional network was identified by OrT/CVA of onand off-stimulation scan pairs acquired in the PD patients during sequence learning. This spatial covariance pattern (Table 2) was characterized by stimulation-related increases (Fig. $2 A$, red) in the right lateral cerebellum (lobule VII) and parahippocampal gyrus (BA 36) and in the left dorsal premotor (BA 6) and inferior parietal (BA 40) regions. These changes were associated with stimulationrelated reductions medially (Fig. $2 \mathrm{~A}$, blue) in the orbitofrontal cortex (BA 11) and caudal supplementary motor area (SMA).

Network activity values (Fig. $2 B$ ) measured in LEARN scans acquired at baseline (OFF) and during stimulation (DBS) exhibited a significant monotonic trend across conditions ( $p=0.008$, permutation test) with increasing pattern expression in 11 of 12 trials. Pattern expression was computed prospectively in the LEARN and MOVE scans from the healthy control subjects. In this group, network values computed in LEARN (Fig. $2 C$, left) were found to be higher than in MOVE ( $p=0.02$, paired Student's $t$ test). In contrast, PD patients scanned at baseline (Fig. $2 C$, right) exhibited reduced network activity in LEARN relative to MOVE $(p<0.005)$. Indeed, learning-specific network responses (i.e., difference between LEARN and MOVE subject scores) for the PD patients in the untreated condition and the healthy control subjects were significantly different between the two groups $\left(F_{(1,16)}=11.2, p<0.005\right.$; group $\times$ task interaction effects). Network activity values computed in the resting condition in the baseline PD scans were also lower than the corresponding values from the healthy control subjects $(p<0.05)$. In both the control and PD groups (Fig. $2 D, E)$, network activity correlated with concurrent measurements of task performance obtained during each LEARN trial (NL, $r=0.84, p<0.01$; PD (baseline), $r=0.74, p=0.03$, Pearson's product moment correlations). 
A LEARN
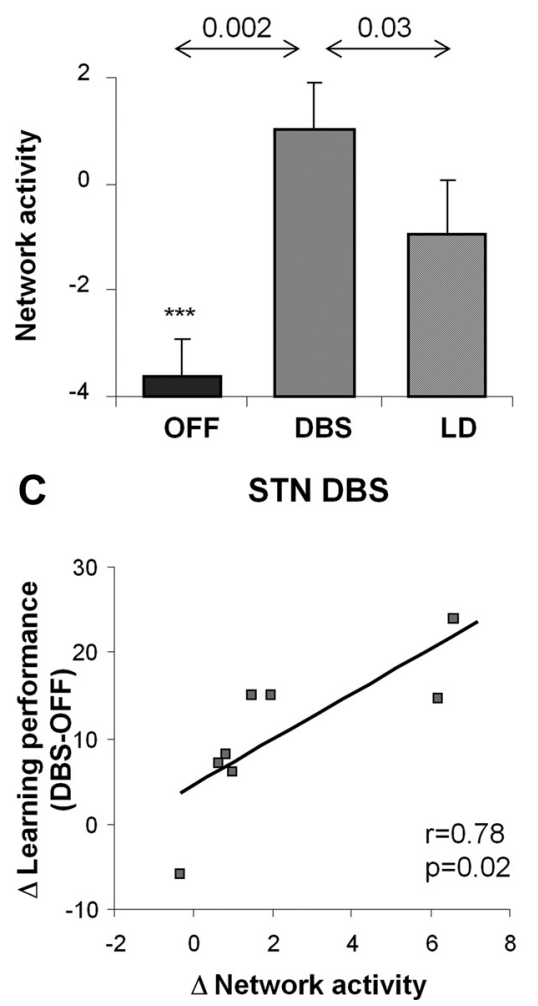

B
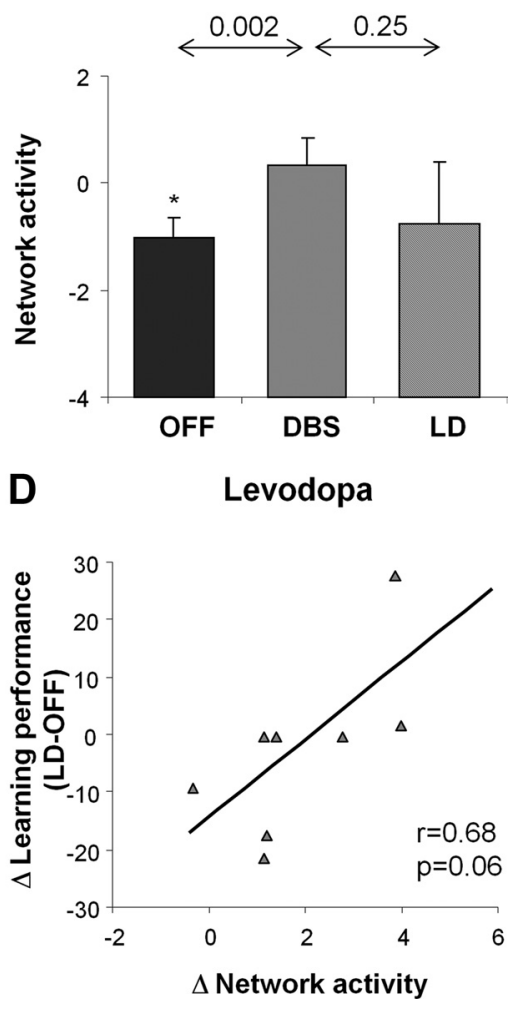

Figure 3. Changes in network activity during treatment. $\boldsymbol{A}$, Measurements of network activity acquired during sequence learning were higher during STN stimulation compared with both the levodopa $(p<0.05)$ and the baseline experimental conditions $(p<0.005)$. $B$, Network activity values measured during motor execution were higher during STN stimulation compared with baseline $(p<0.005)$ but did not differ significantly from values measured during treatment $(p=0.25)$. $C$, Changes in network activity with STN stimulation correlated with concurrent changes in learning performance $(r=0.78, p<0.05)$. D, A correlation of borderline significance $(r=0.68, p=0.06$ ) was present between the levodopa-mediated changes in network activity and concurrent changes in task performance. ${ }^{*} p<0.05,{ }^{* *} p<0.001$ relative to baseline values from healthy control subjects.

\section{Changes in network activity during treatment}

STN stimulation was associated with significant increases in network activity (Fig. $3 A, B$ ) measured during the sequence learning and motor execution tasks $(p<0.002$, paired Student's $t$ tests for separate DBS vs OFF comparisons of network values computed in LEARN and MOVE scans). Nonetheless, stimulation-mediated changes in network activity were greater during learning than during movement (task $\times$ condition interaction: $F_{(1,20)}=4.8, p=0.04,2 \times 2$ RMANOVA). Indeed, individual differences in the degree of network modulation that occurred with stimulation [(LEARN MOVE $)_{\text {DBS }}-(\text { LEARN - MOVE })_{\mathrm{OFF}}$ (Fig. 3C) correlated with concurrent changes in learning performance observed in the same subjects ( $r=0.78, p=0.02$, Pearson's correlation). The stimulation-mediated network changes did not, however, correlate with differences in the DBS parameters used during imaging $(p>0.26)$.

Despite comparable degrees of improvement in clinical motor ratings, levodopa infusion and STN stimulation differed in their impact on learning-related network activity. Network activity measured during learning (Fig. $3 A$ ) was greater for STN stimulation than levodopa treatment ( $p=0.03$, paired Student's $t$ test for DBS vs LD comparison of network values measured in LEARN scans). Significant between-intervention differences in network modulation (Fig. 3B) were not evident in the non-learning tasks (MOVE, $p=$ 0.25 ; REST, $p=0.49)$. A correlation of marginal significance $(r=$
0.68, $p=0.06$ ) was noted between levodopa-mediated differences in network activity measured during learning and concurrent changes in task performance (Fig. $3 D)$.

\section{Nodal analysis}

We next examined the regional correlates of the observed network changes. First, we examined rCBF values in regions with increases in learning-related activity during stimulation (Fig. 2A, red-yellow; Table $2)$. Significant effects of treatment on LEARN scan $\mathrm{rCBF}(p<0.05$, paired Student's $t$ tests) were evident in the left cerebellar hemisphere (lobule VII) and in the right premotor cortex (Fig. 4, top panels). Baseline rCBF values measured in both these areas were lower than normal in the PD patients, regardless of task (cerebellum: LEARN, $p<0.01$; MOVE, $p<0.05$; REST, $p<0.05$; premotor cortex: LEARN, $p<0.01$; MOVE, $p<0.05$; REST, $p<0.01$, Student's $t$ tests). In both regions, $\mathrm{rCBF}$ values were increased by stimulation during learning (DBS > OFF: $p<0.05$, paired Student's $t$ tests for LEARN scan comparisons) but not during motor execution (cerebellum, $p=$ 0.054 ; premotor cortex, $p=0.18$ for MOVE scan comparisons) or rest $(p>$ 0.55 for REST scan comparisons). rCBF values in these regions did not differ significantly across the two interventions (LEARN, $p>0.09$; MOVE, $p>0.37$; REST, $p>0.56$, paired Student's $t$ tests for comparisons of DBS and LD).

Similarly, we examined the $\mathrm{rCBF}$ data from regions with reductions in learning-related activity during stimulation (Fig. 2 A, blue-green; Table 2). Significant treatment effects on rCBF values measured during learning were evident in the orbitofrontal cortex (BA 11) and the SMA (Fig. 5A,B). In both regions, $\mathrm{rCBF}$ values measured in LEARN scans were abnormally elevated in the baseline condition (orbitofrontal cortex, $p<0.05$; SMA, $p<0.01$, Student's $t$ tests). In the orbitofrontal cortex, baseline rCBF measured during movement and rest did not differ from control values $(p>0.65)$. In this region, stimulation was associated with reduced $\mathrm{rCBF}$ during learning $(p<$ 0.04 , paired Student's $t$ test). Significant changes in rCBF during stimulation were not present in this region when measured during movement or rest $(p>0.67)$. Likewise, orbitofrontal rCBF values measured during each of the three tasks did not differ for the two interventions ( $p>0.51$, paired Student's $t$ tests for DBS vs LD comparisons).

In contrast, in the SMA, abnormal elevations in baseline rCBF values were present during learning and also during the performance of the non-learning movement and rest tasks (MOVE, $p<$ 0.05; REST, $p<0.001$, Student's $t$ tests for comparisons with normal values). STN stimulation resulted in significant reductions in SMA values measured during LEARN $(p<0.02)$ and REST $(p<0.01)$; a trend-level reduction $(p=0.1)$ was discerned during MOVE. Indeed, in this region, significant betweenintervention differences were evident during learning and rest 
(LEARN, $p<0.02$; REST, $p<0.003$, paired Student's $t$ tests for comparisons of DBS and LD), with lower rCBF during STN stimulation compared with levodopa. A borderline difference between interventions $(p=0.07)$ was noted in SMA for rCBF values measured during movement. Thus, significant baseline rCBF elevations were evident in the SMA, independent of task. These abnormalities were modulated toward normal by anti-parkinsonian treatment with STN stimulation but not levodopa.

Last, we assessed the consistency of the stimulation effects on rCBF values measured at each node with the concurrent changes observed at the network level (see Materials and Methods) (Fig. 6). Of the four nodal regions with significant stimulation-mediated rCBF changes, only the SMA exhibited local responses that consistently paralleled the network trend. Specifically, rCBF values in this region were remarkable for the presence of only a single violation in the 12 stimulation trials that were performed during learning $(p=$ 0.01 , binomial test, corrected for multiple comparisons). This contrasted with the other nodes (the cerebellum, the premotor cortex, and the orbitofrontal cortex) in which four to five exceptions were noted per region, which did not differ from the number expected by chance $(p>0.48)$.

Despite the presence of significant rCBF changes during treatment in nodal regions, these regional measures did not correlate with concurrent changes in learning performance $(p>0.16)$.

\section{Discussion}

Sequence learning network associated with STN stimulation

Electrophysiological and functional imaging studies have consistently linked the learning of motor sequences to the activation of cortico-striato-pallido-thalamocortical and cerebello-thalamo-cortical neural pathways (Doyon et al., 2009; Orban et al., 2010). Indeed, we have found previously that sequence learning performance was associated with the activation of discrete brain networks in healthy subjects and in unmedicated PD patients (Nakamura et al., 2001; Carbon et al., 2003; Carbon and Eidelberg, 2006). Moreover, changes in learning network activity during the treatment of PD motor symptoms with GPi stimulation or levodopa administration correlated with concurrent recordings of task performance during imaging (Carbon and Eidelberg, 2002; Carbon et al., 2003). That said, these previous studies did not address the possibility of distinct treatment-
A

Cerebellum (Lob. VII) $[-34,-54,-50]$

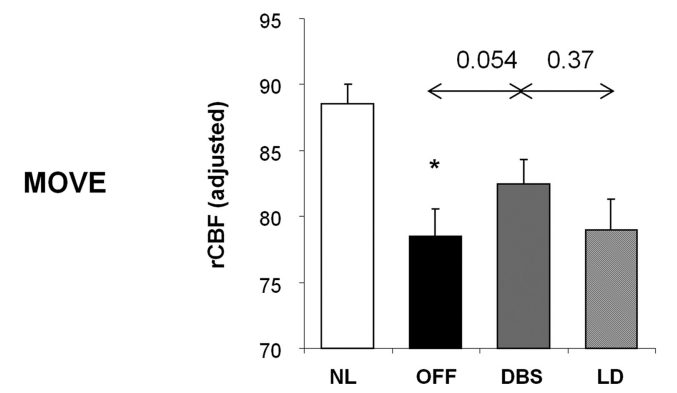

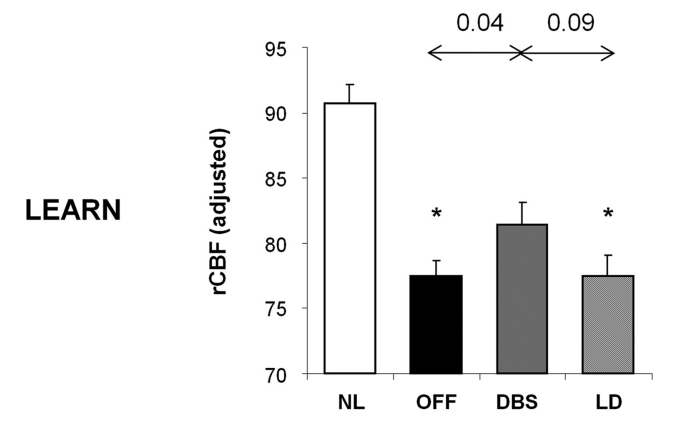

B Premotor cortex (BA6) $[32,20,58]$
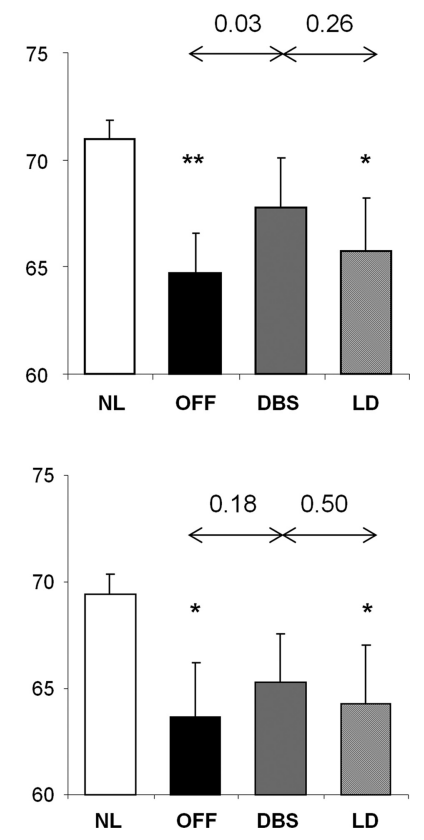

Figure 4. Network regions with increased learning-related activity during stimulation. At the positive (increasing) nodes of the network (Fig. 2, red) represented by the right lateral cerebellum $(\boldsymbol{A})$ and the left dorsal premotor cortex $(\boldsymbol{B})$, baseline rCBF was significantly lower than NL during both sequence learning (LEARN, top) and motor execution (MOVE, bottom) task performance $\left({ }^{*} p<0.05\right.$, ${ }^{* *} p<0.01$, paired Student's $t$ tests). In both regions, STN stimulation increased rCBF values measured during learning $(p<0.05)$ but not when measured during motor execution $(p>0.05)$. rCBF values for the two regions measured during either task did not differ for the two anti-parkinsonian interventions $(p>0.09)$.

\section{A}
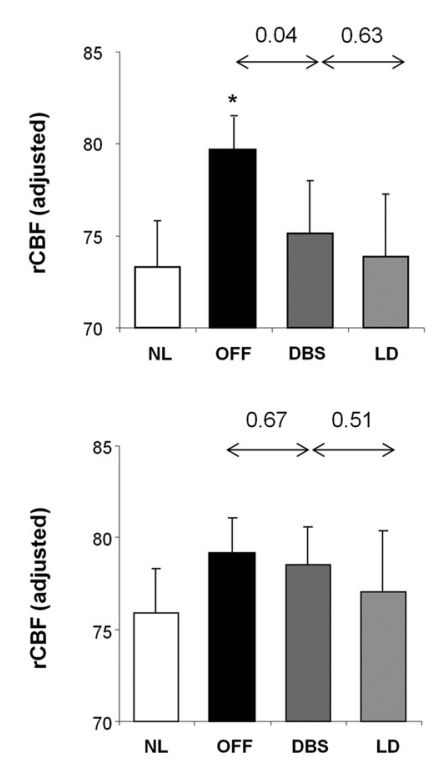

B Supplementary Motor Area (SMA) $[0,-18,68]$
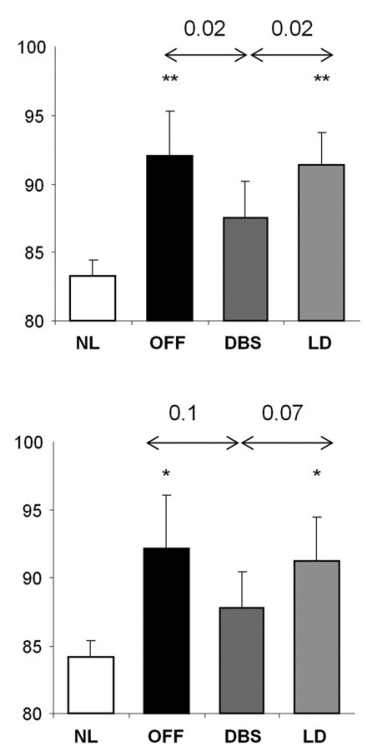

Figure 5. Network regions with declining learning-related activity during stimulation. The negative (declining) nodes of the network (Fig. 2, blue) were localized to the orbitofrontal cortex and the SMA. $A$, In the orbitofrontal region, baseline rCBF values were higher than normal when measured during sequence learning $\left({ }^{*} p<0.05\right.$; top) but not during motor execution ( $p=0.65$; bottom). In this region, rCBF values measured during learning were reduced by STN stimulation $(p<0.05)$ but did not change during motor execution $(p=0.67)$.rCBF values measured during both tasks did not differ across interventions $(p>0.51) . B$, In the SMA, in contrast, baseline rCBF values were higher than normal during both sequence learning and motor execution $\left({ }^{*} p<0.05,{ }^{* *} p<0.01\right)$. STN stimulation was associated with a decline in $\mathrm{rCBF}$ measured during sequence learning $(p<0.05)$ but not during motor execution. Between-intervention rCBF differences in this region were significant when recorded during sequence learning $(p<0.05)$ and at rest $(p<0.005$; data not displayed); marginal differences $(p=0.07)$ were evident when recorded during motor execution. 
A
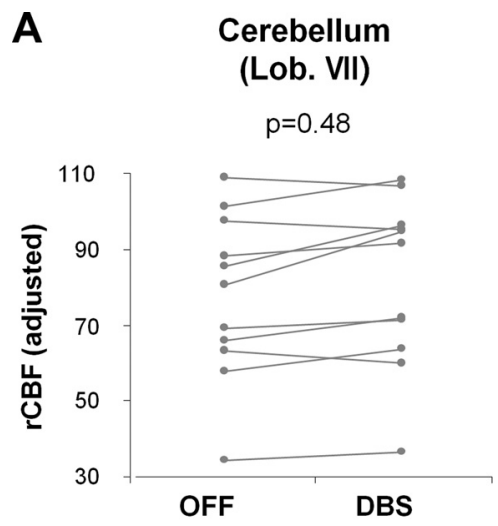

C

Orbitofrontal cortex (BA11)

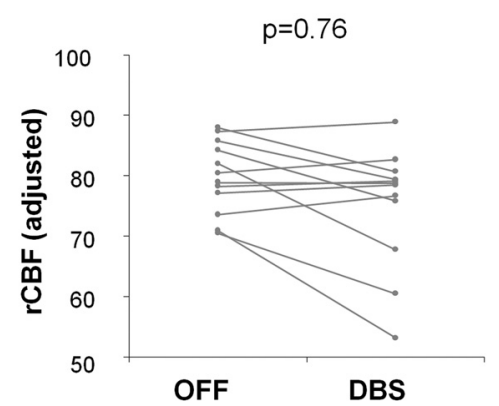

B

Premotor cortex
(BA6)

$p=0.48$
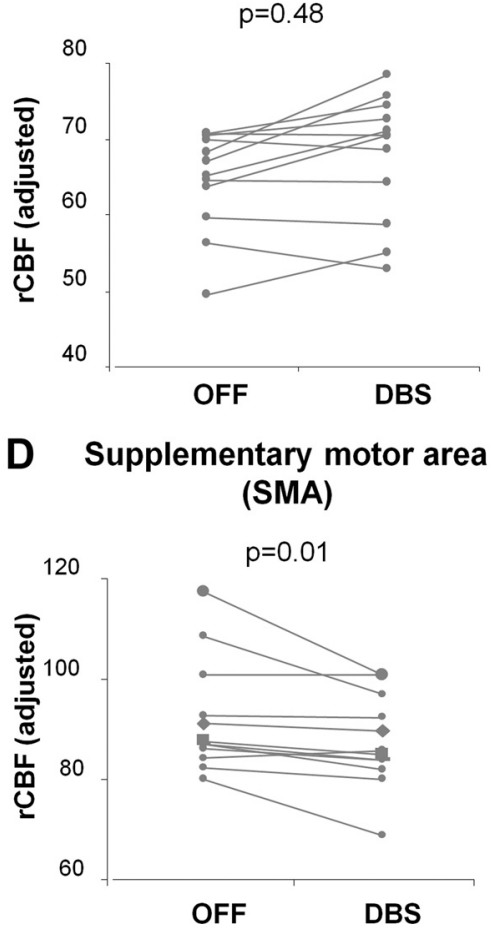

Figure 6. Effects of STN stimulation on neural activity measured in network regions: analysis of individual trials. The effects of STN stimulation on rCBF values measured during learning were evaluated for each of the major network nodes. $A-C$, In the cerebellum, premotor cortex, and orbitofrontal region, the number of exceptions to the network-level trend observed in the on- off stimulation trials conducted during learning ( $4-5$ violations $/ 12$ trials) did not differ from chance $(p>0.48$, binomial tests, corrected for multiple comparisons). $\boldsymbol{D}$, In contrast, in the SMA, there was only a single exception to the network trend (1 violation/12 trials), which differed significantly from chance $(p=0.01)$. This node was therefore considered to be the most consistent and influential of the network regions.

specific network effects on learning pathways.

In the present study, we used OrT/CVA, a multivariate algorithm designed to detect and quantify functional brain networks with monotonically changing subject expression across experimental conditions on an individual case basis (Habeck et al., 2005). This analytical approach differed from that used in our previous studies of learning-related activation patterns in $\mathrm{PD}$ patients (Nakamura et al., 2001; Carbon et al., 2003). We initially applied spatial covariance analysis to the subtracted images (LEARN - MOVE) from healthy subjects (or from untreated PD patients) to identify patterns correlating with learning performance. The resulting learning-related patterns were then used prospectively to quantify the effects of treatment on network activity in individual subjects. This strategy, however, relied on the premise that therapeutic interventions such as DBS act by modulating the activity of normal brain networks (Carbon and Eidelberg, 2002) as opposed to inducing a distinctive set of network changes related specifically to treatment. To address the latter possibility, OrT/CVA was used in the current study to indentify a specific functional network characterized by stimulation-mediated changes. A number of steps were taken to ensure that the resulting network was learning related and not a manifestation of nonspecific stimulation effects on rCBF. First, the network identification procedure (as well as the prospective single scan computations) was conducted in a subspace of the mean centered subject $X$ voxel covariance matrix that was independent of (orthogonal to) that originally specified in relation to motor activation in normal individuals (Carbon et al., 2010a) and that was dem- onstrated in the current cohort of $\mathrm{PD}$ and healthy control subjects. Furthermore, network activity values computed in the baseline scans of the PD patients and in the healthy control subjects correlated significantly with concurrent measurements of learning performance. Last, network values computed in the on-stimulation condition did not correlate with individual differences in the DBS tuning parameters, nor did the latter influence the slope of the observed network-performance correlation line. Thus, although the network was identified using scan pairs obtained off- and onstimulation, the relevant topography was specified by applying OrT/CVA to images acquired during learning and therefore related preferentially to this cognitive process.

\section{Anatomical and functional basis for the learning-related network}

Many of the areas identified as nodes of the learning-related network identified in this study have been described previously in univariate analyses of PET and fMRI activation data from cohorts of healthy subjects performing a variety of sequence learning tasks (for review, see Carbon and Eidelberg, 2006; Doyon et al., 2009; Wilkinson et al., 2009). Typically, the cerebellum, cingulate, premotor cortex, and inferior parietal area are activated in the early phases of learning. In contrast, the striatum, SMA, prefrontal cortex, and precuneus are activated during the later stages of this cognitive process. In the current study, the patients were scanned comparatively early in the learning process, which accords with the areas of increased activity featured by the network. Overall, these nodal regions exhibited abnormally low neural activity in the baseline condition, regardless of the task performed. Nevertheless, the normalization of rCBF that was observed in these areas during stimulation was specific for sequence learning and paralleled the cognitive response to treatment.

At two network nodes, STN stimulation influenced neural activity by lowering abnormal baseline elevations of neural activity. In the orbitofrontal cortex, neural activity was significantly elevated in the baseline scans of the PD patients acquired during learning but not during motor execution or rest. Accordingly, STN stimulation lowered rCBF in this region when measured during learning but effected no change in movement and rest scans from the same subjects. These findings accord with the notion that the orbitofrontal cortex can be deployed during sequence learning under pathological conditions (Feigin et al., 2006). In this vein, the elevation of learning-related neural activity observed in this region in untreated PD patients may be compensatory. Indeed, normalization of $\mathrm{rCBF}$ in this region by either STN stimulation or levodopa is consistent with this interpretation.

In addition to the orbitofrontal cortex, abnormal baseline rCBF elevations in the PD patients were evident in the SMA proper. Given the prominent contribution of increased SMA activity to the abnormal resting metabolic network characteristic of PD (Eidelberg, 2009; Tang et al., 2010), it is not surprising that 
the baseline $\mathrm{rCBF}$ increases observed in this region were task independent. Moreover, STN stimulation improved these regional abnormalities in a similar task-independent manner, with significant reductions in $\mathrm{rCBF}$ during learning and rest and a trend in that direction during movement.

The dominant role of the SMA in mediating the changes in network activity seen during STN stimulation is underscored by the uniform $\mathrm{rCBF}$ treatment responses that characterized this brain region. These findings are consistent with previous data from human subjects and experimental animal models. A recent resting-state fMRI study demonstrated a significant increase in the functional connectivity of pathways linking the STN and cortical motor regions (including SMA) in PD patients (Baudrexel et al., 2011). Indeed, abnormal elevations in the metabolic activity of the STN and motor cortex (M1/SMA) have been observed during PD progression (Huang et al., 2007), in parallel with increases in the PD-related metabolic network mentioned above (Lin et al., 2008; Eidelberg, 2009; Tang et al., 2010). These changes are likely attributable to overactivity of the "hyperdirect" M1/ SMA-STN projection system demonstrated in animal models (Dejean et al., 2008; Nachev et al., 2008). Oversynchronization of high-frequency oscillatory activity in this loop may account for the abnormally high cerebral blood flow and metabolic activity seen in these regions in untreated PD patients. In this context, STN stimulation lowered these values toward normal, with concomitant improvement in learning performance as well as generalized motor functioning. The current data are also compatible with the notion that high-frequency STN DBS exerts its therapeutic effect through antidromic modulation of cortical-STN axonal projections (Gradinaru et al., 2009). In the rodent, localized modulation of motor cortical activity may be needed for the therapeutic effects of STN stimulation to occur. Nonetheless, in human PD patients, clinical outcome is likely to be contingent on changes in activity of the network as a whole. Indeed, concurrent normalization of learning-related $\mathrm{rCBF}$ abnormalities at the other network nodes (e.g., cerebellum, premotor cortex, and inferior parietal area) argues in favor of a combination of stimulation-mediated functional effects at multiple sites.

One may speculate as to why analogous therapeutic effects on learning performance and associated network activity were not observed when levodopa was administered to the same subjects. Prospective quantification of pattern expression in scans acquired during levodopa infusion suggested that the learningrelated network was indeed deployed in this treatment condition. Nonetheless, on average, network activity was significantly lower with levodopa than the motorically equivalent stimulation condition. In this vein, subject scores for the network measured in LEARN scans acquired during levodopa infusion were on average $1 \mathrm{SD}$ below the normal mean, whereas corresponding values measured during STN stimulation were $\sim 1 \mathrm{SD}$ above normal. Of note, there was no correlation between clinical descriptors, such as subject age, symptom duration, and motor severity ratings, and treatment-mediated changes in learning performance and associated network activity.

Analogous trends were also seen at the regional level: STN stimulation was relatively more effective than levodopa in normalizing local neural activity in network areas. This difference was most pronounced at the critical SMA node. It is likely that modulation of abnormal baseline activity in this region by STN stimulation - and the accompanying improvement in learning performance-stems from the selectivity of this intervention for the axons of the hyperdirect M1/SMA-STN pathway. That functional changes in this pathway were seen in response to cognitive as well as motor demands suggests a potential role for the SMA as a discrete treatment target. Indeed, therapeutic interventions directed specifically at this brain region may benefit PD patients with medication-refractory symptoms, including commonly encountered cognitive deficits in sequence learning and skill acquisition (Ghilardi et al., 2003; Carbon and Eidelberg, 2006; Doyon, 2008). Importantly, the findings also lend support to the idea of conceptualizing the therapeutic target as the entire functional network as a single entity, as opposed to each of its regional elements in isolation.

\section{References}

Aarsland D, Brønnick K, Larsen JP, Tysnes OB, Alves G (2009) Cognitive impairment in incident, untreated Parkinson disease: the Norwegian ParkWest study. Neurology 72:1121-1126.

Aarsland D, Bronnick K, Williams-Gray C, Weintraub D, Marder K, Kulisevsky J, Burn D, Barone P, Pagonabarraga J, Allcock L, Santangelo G, Foltynie T, Janvin C, Larsen JP, Barker RA, Emre M (2010) Mild cognitive impairment in Parkinson disease: a multicenter pooled analysis. Neurology 75:1062-1069.

Argyelan M, Carbon M, Ghilardi MF, Feigin A, Mattis P, Tang C, Dhawan V, Eidelberg D (2008) Dopaminergic suppression of brain deactivation responses during sequence learning. J Neurosci 28:10687-10695.

Asanuma K, Tang C, Ma Y, Dhawan V, Mattis P, Edwards C, Kaplitt MG, Feigin A, Eidelberg D (2006) Network modulation in the treatment of Parkinson's disease. Brain 129:2667-2678.

Baudrexel S, Witte T, Seifried C, von Wegner F, Beissner F, Klein JC, Steinmetz H, Deichmann R, Roeper J, Hilker R (2011) Resting state fMRI reveals increased subthalamic nucleus-motor cortex connectivity in Parkinson's disease. Neuroimage 55:1728-1738.

Beck A (1987) Beck Depression Inventory: Manual. San Antonio, TX: Psychological Corporation.

Benedict RH, Schretlen D, Groninger L, Brandt J (1998) Hopkins Verbal Learning Test—revised: normative data and analysis of interform and test-retest reliability. Clin Neuropsychol 12:43-55.

Benton A, Hamsher K, Varyney N, Spreen O (1983) Contributions to neuropsychological assessment. New York: Oxford UP.

Blair JR, Spreen O (1989) Predicting premorbid IQ: a revision of the National Adult Reading Test. Clin Neuropsychol 3:129-136.

Brandt J (1991) The Hopkins Verbal Learning Test: development of a new verbal memory rest with six alternate forms. Clin Neuropsychol 5:125-142.

Carbon M, Eidelberg D (2002) Modulation of regional brain function by deep brain stimulation: studies with positron emission tomography. Curr Opin Neurol 15:451-455.

Carbon M, Eidelberg D (2006) Functional imaging of sequence learning in Parkinson's disease. J Neurol Sci 248:72-77.

Carbon M, Ghilardi MF, Feigin A, Fukuda M, Silvestri G, Mentis MJ, Ghez C, Moeller JR, Eidelberg D (2003) Learning networks in health and Parkinson's disease: reproducibility and treatment effects. Hum Brain Mapp 19:197-211.

Carbon M, Argyelan M, Habeck C, Ghilardi MF, Fitzpatrick T, Dhawan V, Pourfar M, Bressman SB, Eidelberg D (2010a) Increased sensorimotor network activity in DYT1 dystonia: a functional imaging study. Brain 133:690-700.

Carbon M, Reetz K, Ghilardi MF, Dhawan V, Eidelberg D (2010b) Early Parkinson's disease: longitudinal changes in brain activity during sequence learning. Neurobiol Dis 37:455-460.

Cools R, Altamirano L, D'Esposito M (2006) Reversal learning in Parkinson's disease depends on medication status and outcome valence. Neuropsychologia 44:1663-1673.

Dejean C, Gross CE, Bioulac B, Boraud T (2008) Dynamic changes in the cortex-basal ganglia network after dopamine depletion in the rat. J Neurophysiol 100:385-396.

Doyon J (2008) Motor sequence learning and movement disorders. Curr Opin Neurol 21:478-483.

Doyon J, Bellec P, Amsel R, Penhune V, Monchi O, Carrier J, Lehéricy S, Benali H (2009) Contributions of the basal ganglia and functionally related brain structures to motor learning. Behav Brain Res 199:61-75.

Efron B, Tibshirani RJ (1994) An Introduction to the Bootstap. New York: CRC. 
Eidelberg D (2009) Metabolic brain networks in neurodegenerative disorders: a functional imaging approach. Trends Neurosci 32:548-557.

Fahn S, Elton, RL, Members of the UPDRS Development Committee (1987) Unified Parkinson's Disease Rating Scale. In: Recent developments in Parkinson's disease (Fahn S, Marsden DM, Goldstein M, Calne DB, eds), pp 153-163. New York: MacMillan.

Feigin A, Ghilardi MF, Carbon M, Edwards C, Fukuda M, Dhawan V, Margouleff C, Ghez C, Eidelberg D (2003) Effects of levodopa on motor sequence learning in Parkinson's disease. Neurology 60:1744-1749.

Feigin A, Ghilardi MF, Huang C, Ma Y, Carbon M, Guttman M, Paulsen JS, Ghez CP, Eidelberg D (2006) Preclinical Huntington's disease: compensatory brain responses during learning. Ann Neurol 59:53-59.

Frank MJ, Claus ED (2006) Anatomy of a decision: striato-orbitofrontal interactions in reinforcement learning, decision making, and reversal. Psychol Rev 113:300-326.

Fukuda M, Ghilardi MF, Carbon M, Dhawan V, Ma Y, Feigin A, Mentis MJ, Ghez C, Eidelberg D (2002) Pallidal stimulation for parkinsonism: improved brain activation during sequence learning. Ann Neurol 52:144-152.

Ghilardi MF, Eidelberg D, Silvestri G, Ghez C (2003) The differential effect of PD and normal aging on early explicit sequence learning. Neurology 60:1313-1319.

Ghilardi MF, Feigin AS, Battaglia F, Silvestri G, Mattis P, Eidelberg D, Di Rocco A (2007) L-Dopa infusion does not improve explicit sequence learning in Parkinson's disease. Parkinsonism Relat Disord 13:146-151.

Ghilardi MF, Moisello C, Silvestri G, Ghez C, Krakauer JW (2009) Learning of a sequential motor skill comprises explicit and implicit components that consolidate differently. J Neurophysiol 101:2218-2229.

Ghilardi M, Ghez C, Dhawan V, Moeller J, Mentis M, Nakamura T, Antonini A, Eidelberg D (2000) Patterns of regional brain activation associated with different forms of motor learning. Brain Res 871:127-145.

Gradinaru V, Mogri M, Thompson KR, Henderson JM, Deisseroth K (2009) Optical deconstruction of parkinsonian neural circuitry. Science 324:354-359.

Habeck C, Stern Y (2010) Multivariate data analysis for neuroimaging data: overview and application to Alzheimer's disease. Cell Biochem Biophys 58:53-67.

Habeck C, Krakauer JW, Ghez C, Sackeim HA, Eidelberg D, Stern Y, Moeller JR (2005) A new approach to spatial covariance modeling of functional brain imaging data: ordinal trend analysis. Neural Comput 17:1602-1645.

Hauser RA, Holford NH (2002) Quantitative description of loss of clinical benefit following withdrawal of levodopa-carbidopa and bromocriptine in early Parkinson's disease. Mov Disord 17:961-968.

Hirano S, Asanuma K, Ma Y, Tang C, Feigin A, Dhawan V, Carbon M, Eidelberg D (2008) Dissociation of metabolic and neurovascular responses to levodopa in the treatment of Parkinson's disease. J Neurosci 28:4201-4209.

Huang C, Tang C, Feigin A, Lesser M, Ma Y, Pourfar M, Dhawan V, Eidelberg D (2007) Changes in network activity with the progression of Parkinson's disease. Brain 130:1834-1846.

Kehagia AA, Barker RA, Robbins TW (2010) Neuropsychological and clinical heterogeneity of cognitive impairment and dementia in patients with Parkinson's disease. Lancet Neurol 9:1200-1213.
Levin BE, Katzen HL (2005) Early cognitive changes and nondementing behavioral abnormalities in Parkinson's disease. Adv Neurol 96:84-94.

Lin TP, Carbon M, Tang C, Mogilner AY, Sterio D, Beric A, Dhawan V, Eidelberg D (2008) Metabolic correlates of subthalamic nucleus activity in Parkinson's disease. Brain 131:1373-1380.

Mattis PJ, Tang CC, Ma Y, Dhawan V, Eidelberg D (2011) Networks correlates of the cognitive response to levodopa in Parkinson's disease. Neurology 77:858-865.

Moeller J, Habeck C (2006) Reciprocal benefits of mass-univariate and multivariate modeling in brain mapping: applications to event-related functional MRI, H215O-, and FDG PET. Int J Biomed Imaging 2006:1-13.

Mure H, Hirano S, Tang CC, Isaias IU, Antonini A, Ma Y, Dhawan V, Eidelberg D (2011) Parkinson's disease tremor-related metabolic network: characterization, progression, and treatment effects. Neuroimage 54:1244-1253.

Nachev P, Kennard C, Husain M (2008) Functional role of the supplementary and pre-supplementary motor areas. Nat Rev Neurosci 9:856-869.

Nakamura T, Ghilardi MF, Mentis M, Dhawan V, Fukuda M, Hacking A, Moeller JR, Ghez C, Eidelberg D (2001) Functional networks in motor sequence learning: abnormal topographies in Parkinson's disease. Hum Brain Mapp 12:42-60.

Nelson HE (1982) The National Adult Reading Test (NART): Test Manual. Windsor, UK: NFER-Nelson.

Orban P, Peigneux P, Lungu O, Albouy G, Breton E, Laberenne F, Benali H, Maquet P, Doyon J (2010) The multifaceted nature of the relationship between performance and brain activity in motor sequence learning. Neuroimage 49:694-702.

Parsons TD, Rogers SA, Braaten AJ, Woods SP, Tröster AI (2006) Cognitive sequelae of subthalamic nucleus deep brain stimulation in Parkinson's disease: a meta-analysis. Lancet Neurol 5:578-588.

Schmahmann JD, Doyon J, Toga AW, Petrides M, Evans AC (2000) MRI atlas of the human cerebellum. San Diego: Academic.

Schretlen D (1989) Brief test of attention. Odessa, FL: Psychological Assessment Resources.

Smith A (1982) Symbol Digit Modalities Test (SDMT) Manual (revised). Los Angeles: Western Psychological Services.

Spetsieris PG, Eidelberg D (2011) Scaled subprofile modeling of resting state imaging data in Parkinson's disease: methodological issues. Neuroimage 54:2899-2914.

Tang CC, Poston KL, Dhawan V, Eidelberg D (2010) Abnormalities in metabolic network activity precede the onset of motor symptoms in Parkinson's disease. J Neurosci 30:1049-1056.

Wilkinson L, Khan Z, Jahanshahi M (2009) The role of the basal ganglia and its cortical connections in sequence learning: evidence from implicit and explicit sequence learning in Parkinson's disease. Neuropsychologia 47:2564-2573.

Williams AE, Arzola GM, Strutt AM, Simpson R, Jankovic J, York MK (2011) Cognitive outcome and reliable change indices two years following bilateral subthalamic nucleus deep brain stimulation. Parkinsonism Relat Disord 17:321-327.

Williams-Gray CH, Foltynie T, Brayne CE, Robbins TW, Barker RA (2007) Evolution of cognitive dysfunction in an incident Parkinson's disease cohort. Brain 130:1787-1798. 